TERRA. Revista de Desarrollo Local e-ISSN: 2386-9968

Número 9 (2021), 147-159

DOI 10.7203/terra.9.21400

IIDL - Instituto Interuniversitario de Desarrollo Local

\title{
Protocolo de buenas prácticas de bioseguridad frente al COVID19: los operadores turísticos de la localidad de Usme (Bogotá, Colombia)
}

\author{
Esperanza Padilla Murcia \\ Investigación Grupo Salud y Ambiente. Politécnico Internacional. Colombia. \\ esperanza.padilla@pi.edu.co

\section{Esthella Bonza Quesada} \\ Investigación Grupo Gastronomía y Turismo. Politécnico Internacional. Colombia. \\ esthella.bonza@pi.edu.co
}

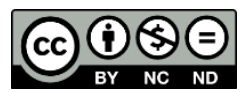

Esta obra se distribuye con la licencia Creative Commons Reconocimiento-NoComercial-SinObraDerivada 4.0 Internacional 


\section{SECCIÓN EXPERIENCIAS Y BUENAS PRÁCTICAS}

\section{Protocolo de buenas prácticas de bioseguridad frente al COVID19: los operadores turísticos de la localidad de Usme (Bogotá, Colombia)}

Resumen: La pandemia del COVID-19 obligó a todos los países del mundo a tomar medidas frente al tema de la bioseguridad para prevenir y mitigar los riesgos de contagio de las personas en las viviendas, las organizaciones y en los territorios. Lo anterior incluyó al sector del turismo, donde los prestadores de servicio tuvieron que elaborar los correspondientes protocolos para poder funcionar con buenas prácticas. Es así como el objetivo de este estudio fue construir un protocolo de buenas prácticas de bioseguridad COVID-19 para cuatro operadores del turismo comunitario de la localidad de Usme en Bogotá-Colombia. La metodología de investigación utilizada fue el estudio de caso cualitativo con el que se empleó la encuesta como instrumento de recolección. Entre los resultados obtenidos está la descripción de las características de los cuatro operadores turísticos, la identificación y comparación de la legislación correspondiente, y la co-construcción del documento de bioseguridad. Como conclusión principal, se determinó que el documento de buenas prácticas de bioseguridad para los operadores turísticos de la localidad de Usme debe responder principalmente a servicios turísticos como: reserva natural, realización de caminatas ecológicas, avistamiento de aves, actividades de finca, gastronomía y alojamiento tipo camping, lo que exige tener en cuenta las buenas prácticas especificadas en las resoluciones 666, 1421,1285, 1059 y 749, todas de 2020.

Palabras clave: Medioambiente, bioseguridad, legislación, sostenibilidad, turismo.

Recibido: 28 de julio de 2021

Devuelto para revisión: -

Aceptado: 23 de septiembre de 2021

Referencia / Citation:

Padilla, E., y Bonza, E. (2021). Protocolo de buenas prácticas de bioseguridad frente al COVID19: los operadores turísticos de la localidad de Usme (Bogotá, Colombia). TERRA. Revista de Desarrollo Local, (9), 147-159. DOI 10.7203/terra.9.21400 


\section{INTRODUCCIÓN}

El pasado 11 de marzo de 2020, la Organización Mundial de la Salud (OMS) declaró pandemia global por el brote del virus COVID-19. En ese sentido, todos los países se vieron en la obligación de tomar medidas en bioseguridad, lo que implicó el desarrollo de acciones preventivas para eliminar o minimizar el virus, que puede afectar la salud de las personas (Resolución 666, 2020). Estas medidas de protección, en el caso de Colombia, se acordaron mediante la resolución 666 de 2020, como norma general para todos los sectores, incluido el sector turístico. Sin embargo, en dicho sector económico también se originaron otras resoluciones con protocolos de bioseguridad específicos para actividades relacionadas con reservas naturales, áreas y atractivos turísticos; temas de alojamiento, gastronomía, comercio y servicio de alimentos, entre otros.

Lo anterior generó, en los diferentes operadores turísticos, la necesidad de elaborar el protocolo de buenas prácticas de bioseguridad COVID-19, como fue para el caso del 1) Agroparque Los Soches (APS), 2) la Granja de Mary (GM), 3) Mujeres Chucuas (MC) y 4) Mutar (M), cuatro operadores rurales de una comunidad de la localidad de Usme en Bogotá, Colombia. Es en esta en donde se desarrolló la presente investigación, compuesta por una comunidad rural que encontró en el turismo comunitario la forma de emprender y generar progreso para los locales a través de la reserva natural y los servicios complementarios que ofertan, y que se vieron afectados debido a los cierres generados por la pandemia como parte de las medidas preventivas tomadas por los entes gubernamentales, y que a su vez se autorizaba su reapertura con la aprobación de un protocolo de bioseguridad. Para ello, dicha comunidad solicitó a la Institución de Educación Superior (IES), el apoyo para la construcción del correspondiente documento técnico. De allí, el grupo de investigación salud y medio ambiente junto con el grupo de gastronomía y turismo de la IES asumieron el reto de este caso a partir de un trabajo interdisciplinario, y desde un enfoque teórico del turismo comunitario. Este último entendido como toda organización, oferta u estrategia empresarial y turística que busca aprovechar el patrimonio natural y cultural de una comunidad para favorecer su desarrollo y bienestar (Maldonado, 2005; Ministerio de Comercio, Industria y Turismo -MCIT-, 2012; Cardona y Burgos, 2015).

Como respuesta a la solicitud generada por la comunidad, y para atender las medidas de bioseguridad necesarias para el desarrollo de las diferentes actividades turísticas prestadas por los operadores turísticos del Agroparque, se procedió a desarrollar el proyecto de investigación cuyo objetivo fue diseñar el protocolo de buenas prácticas de bioseguridad para cuatro operadores turísticos en la localidad de Usme, que permitiera reactivar la operación post COVID 19 y fortalecer el turismo comunitario en el Agroparque Los Soches. Para lograr el objetivo se plantó responder a la siguiente pregunta de investigación: ¿cómo construir un protocolo de buenas prácticas de bioseguridad COVID19 para cuatro operadores turísticos de la localidad, que responda al contexto normativo del sector turístico a nivel nacional?

Como resultado, se elaboró el presente reporte de caso, el cual tiene como propósito exponer la metodología empleada para la construcción del protocolo de buenas prácticas, los hallazgos obtenidos en el análisis de la legislación generada frente a las características de cada uno de los operadores turísticos y cómo estos se entrelazan con el protocolo correspondiente. 


\section{BASES CONCEPTUALES}

Como elementos teórico-conceptuales para el desarrollo y comprensión de la investigación y el diseño del protocolo de buenas prácticas de bioseguridad se partió de conceptos relacionados con la bioseguridad y el COVID 19, así como, algunos vinculados con el área de turismo y turismo comunitario que se presentan a continuación.

La bioseguridad, se define como el conjunto de medidas preventivas que tienen por objeto eliminar o minimizar el factor de riesgo biológico que pueda llegar a afectar a la salud, el medio ambiente o la vida de las personas, asegurando que el desarrollo o producto final de dichos procedimientos no atenten contra la salud y seguridad de los trabajadores (Resolución 666, 2020, p.6).

El COVID-19, que hace referencia a una enfermedad infecciosa generada por un nuevo coronavirus, que causa una Infección Respiratoria Aguda (IRA), es decir una gripa, que puede ser leve, moderada o severa. Puede producir fiebre, tos, secreciones nasales (mocos) y malestar general (Programa de Bioseguridad Covid-19, 2020, p.8).

Además, se presentan los elementos conceptuales relacionados con el turismo: un área turística: entendida como polígono que delimita el área de certificación y que hace parle de un destino turístico (Programa de Bioseguridad Covid-19, 2020, p.7).

El atractivo turístico: aquellas zonas urbanas o rurales, ecosistemas, paisaje, plazas, vías, monumentos, construcciones y otros, que por sus condiciones y características atrae la atención del turista, y que hacen parte del inventario que para este efecto emita el Ministerio de Comercio, Industria y Turismo (Programa de Bioseguridad Covid-19, 2020, p.7).

El turismo comunitario: se considera como una alternativa empresarial, turística y de desarrollo local, para salvar las disparidades sociales y económicos que adolecen las comunidades rurales, además de una vía que pueden utilizar las comunidades que buscan generar otros ingresos dentro de sus actividades económicas cotidianas, utilizando los recursos culturales, naturales y locales en una región en particular (Maldonado, 2005; MCIT, 2012; Comisión Económica Para América Latina y el Caribe-CEPAL-, 1999, en Cardona y Burgos, 2015, p.33).

Ahora, en Colombia, de acuerdo con los lineamientos de política para el desarrollo del turismo comunitario, el turismo comunitario debe comprenderse como una estrategia local de aprovechamiento de ventajas comparativas en condiciones complejas desde lo social, económico, ambiental y cultural, que con unas mínimas oportunidades y capacidades pueden transformar su realidad o entorno en oportunidades competitivas para las comunidades involucradas, otorgando así soluciones prácticas a problemas complejos como la paz y el desarrollo. También se constituye en una alternativa innovadora de aprovechamiento de los recursos locales de manera sostenible, que permiten generar empleo y nuevas formas de generación de ingresos económicos a comunidades con recursos económicos limitados o excluidas (MCIT, 2012).

Por su parte, el turismo rural se corresponde a tipo de actividad turística en el que la experiencia del visitante está relacionada con un amplio espectro de productos vinculados por lo general con las actividades de naturaleza, la agricultura, las formas de vida y las culturas rurales, la pesca con caña y la visita a lugares de interés (OMS, s.f. Par.1). 


\section{METODOLOGIA}

El enfoque metodológico de esta investigación es un estudio de caso de corte cualitativo interpretativo (Ceballos- Herrera, 2009) de tipo instrumental (Stake, 1998), que pretendió comprender cómo construir un protocolo de buenas prácticas de bioseguridad para prevenir y mitigar el COVID-19 y así favorecer el trabajo turístico de los cuatro operadores indicados previamente en Bogotá-Colombia, dentro del contexto del turismo comunitario.

La legislación existente de los protocolos de bioseguridad COVID-19 para el sector del turismo, como temática de este estudio de caso, describe una situación compleja (Stake, 1998), que responde a la actualidad y a la vida real del sector turístico, como menciona Yin (1985).

De igual modo, este estudio se considera de tipo colectivo (Stake, 1998) debido a que se buscó revisar comparativamente los cuatro casos entre sí, para tratar de detectar patrones de semejanzas y diferencias (Hernández et al., 2014, p.8), tanto en las características de los operadores turísticos como en el contexto normativo correspondiente a los protocolos de bioseguridad COVID-19 para cada uno.

Para construir el protocolo de buenas prácticas de bioseguridad COVID-19 de cuatro operadores turísticos de la localidad de Usme, que respondiera al contexto normativo del sector turístico a nivel nacional, se llevaron a cabo siete pasos (Tabla 1).

La selección de los casos a abordar en este estudio y, en cuanto al paso uno del procedimiento, se puede decir que se presentó de manera coyuntural, es decir, por la necesidad de elaborar el protocolo de bioseguridad COVID-19 ajustado a la legislación vigente por parte de los cuatro operadores turísticos. De allí, se siguió al paso dos, donde se elaboró una carta de compromiso con todos los líderes y participantes de los operadores turísticos, se estableció un plan intensivo de trabajo desde el mes de julio al mes de diciembre de 2020, y se firmó el permiso para poder acceder a toda la información necesaria para la construcción del protocolo en cuestión.

Para el paso tres, se consultó la norma general de bioseguridad para todos los establecimientos (Tabla 3) y de allí se seleccionaron las categorías para elaborar el primer instrumento de recolección, que tendría como objetivo caracterizar a los cuatro operadores turísticos en los aspectos generales requeridos según la norma principal. Así mismo, en el paso tres, se consultaron diferentes documentos y experiencias de protocolos de bioseguridad elaborados por otros sectores, al igual que parámetros establecidos por las autoridades internacionales y nacionales (OMT, 2020; MCIT, 2020; IDT, 2020; Asociación Colombiana de Agencia de Viajes y Turismo, 2020; Politécnico Internacional, 2020).

Como se menciona en el paso cuatro, todos los operadores turísticos se involucraron desde el inicio hasta el final en la construcción del protocolo. Para ello, se realizaron aproximadamente diez reuniones de trabajo y se diligenciaron en la presencialidad, los instrumentos de recolección de información. De manera que en el paso cinco se llevó a cabo una dinámica de trabajo tanto virtual como presencial que permitió la escritura, concertación y socialización del documento final del protocolo de bioseguridad COVID19 para los cuatro operadores turísticos.

No obstante, simultáneamente a los pasos tres y cinco, se fue ejecutando el paso seis, con el que se analizaron los datos recolectados en matrices comparativas. De allí, se extrajeron 
patrones de semejanzas y diferencias entre los cuatro operadores turísticos y entre las normas seleccionadas concernientes a los protocolos de bioseguridad COVID-19 de estos prestadores de servicios turísticos.

Al final, el análisis y el trabajo colaborativo con los participantes permitió la coconstrucción de un solo documento de bioseguridad para los cuatro operadores que hacen parte del turismo comunitario de la localidad de Usme.

Tabla 1. Procedimiento a seguir para el estudio de caso

\begin{tabular}{|c|c|}
\hline Pasos & Actividades a realizar \\
\hline $\begin{array}{l}\text { 1. Identificar y seleccionar } \\
\text { los casos o unidades de } \\
\text { análisis }\end{array}$ & $\begin{array}{l}\text { Solicitud de los operadores turísticos para elaborar el protocolo de } \\
\text { bioseguridad. } \\
\text { Diálogos con los líderes de la comunidad del Agroparque Los Soches. }\end{array}$ \\
\hline $\begin{array}{l}\text { 2. Gestionar y obtener los } \\
\text { permisos de los gatekeepers } \\
\text { de cada operador turístico }\end{array}$ & $\begin{array}{l}\text { Elaboración de carta de compromiso. } \\
\text { Socialización y concertación. } \\
\text { Firma de las partes. }\end{array}$ \\
\hline $\begin{array}{l}\text { 3. Investigar los } \\
\text { antecedentes y su contexto }\end{array}$ & $\begin{array}{l}\text { Recopilación de información acerca de protocolos de bioseguridad y de } \\
\text { antecedentes del sector. } \\
\text { Revisión de la norma general de protocolos de bioseguridad COVID-19 } \\
\text { para todos los establecimientos. } \\
\text { Elaboración de la ficha de caracterización de los prestadores de } \\
\text { servicios con base en la norma general de bioseguridad COVID-19. } \\
\text { Consulta de las normas correspondientes a los prestadores de servicio } \\
\text { turístico involucrados, según características identificadas. } \\
\text { Comparación de las normas a través de una matriz comparativa. } \\
\text { Construcción de una propuesta preliminar de lineamientos del } \\
\text { protocolo. } \\
\text { Construcción del segundo instrumento (diario de campo). }\end{array}$ \\
\hline $\begin{array}{l}\text { 4. Involucrar a los } \\
\text { operadores turísticos }\end{array}$ & $\begin{array}{l}\text { Desde el inicio hasta el final, todos los operadores turísticos estuvieron } \\
\text { involucrados, mucho aún en el trabajo de campo. }\end{array}$ \\
\hline $\begin{array}{l}\text { 5. Realizar trabajo de } \\
\text { campo (tanto virtual como } \\
\text { presencial) }\end{array}$ & $\begin{array}{l}\text { Participación en aproximadamente } 10 \text { reuniones de trabajo. } \\
\text { Concertación de instrumentos de recolección y estructura del protocolo } \\
\text { (trabajada en el paso 3). } \\
\text { Escritura preliminar del protocolo de bioseguridad. } \\
\text { Co-construcción de la estructura final del documento del protocolo de } \\
\text { bioseguridad, según normas y diarios de campo. } \\
\text { Socialización del documento final. }\end{array}$ \\
\hline 6. Analizar los datos & $\begin{array}{l}\text { Comparación de semejanzas y diferencias entre los prestadores de } \\
\text { servicios tal como se ilustra en figura } 1 . \\
\text { Cotejo de las diferentes normas relacionadas con los protocolos de } \\
\text { bioseguridad del sector turismo versus las características de los } \\
\text { prestadores de servicios participantes. }\end{array}$ \\
\hline $\begin{array}{l}\text { 7. Elaborar el reporte } \\
\text { de caso }\end{array}$ & $\begin{array}{l}\text { Descripción } \\
\text { Cuadros y figuras }\end{array}$ \\
\hline
\end{tabular}

Fuente: elaboración propia a partir de Harvard Business School y Design Management Institute (1997), citado por Hernández et al. (2014, p.5), y Ceballos y Herrera (2009). 


\section{RESULTADOS: DISEÑO Y CONSTRUCCIÓN DEL PROTOCOLO DE BUENAS PRÁCTICAS DE BIOSEGURIDAD}

En resumen, se puede afirmar que el protocolo de buenas prácticas de bioseguridad COVID-19 se co-construyó en tres pasos: primero, la caracterización y contextualización de los operadores turísticos; segundo, la identificación y comparación de las normas según las características de los prestadores de servicio turístico; y tercero, la coconstrucción de una estructura integrada del documento de buenas prácticas de bioseguridad teniendo en cuenta las normas reglamentarias para estos operadores.

En cuanto a las características y al contexto de los prestadores de servicio turístico (APS, MC, GM y M), de acuerdo con la Alcaldía de Bogotá (2010), se reconoció que todos están ubicados en la localidad de Usme, costado suroriental de la sabana de Bogotá sobre el kilómetro 0 vía Villavicencio, en el área rural protegida de dicha localidad. Esta área corresponde al $84 \%$ del área total de esta zona (18.306 hectáreas) y se encuentran 15 veredas, entre las cuales está la de Los Soches, con 122 familias y en la que están tres operadores turísticos del caso (APS; MC y GM), y la Vereda de Agualinda Chiguaza, con 450 familias, donde se ubica el cuarto operador (M).

De igual modo, en la comparación de las características se identificaron semejanzas y diferencias en cuanto a la oferta de servicios entre los operadores participantes (Tabla 2). En general se destacan seis servicios: reserva natural, gastronomía, caminatas ecológicas, avistamiento de aves, alojamiento de camping y actividades de finca o granja (Matriz de caracterización de los operadores, 2020). Dichos servicios, desde el turismo comunitario, evidencian el aprovechamiento tanto del patrimonio natural como cultural del territorio, así como la propuesta de una cadena de valor que motiva a los turistas a desplazarse a este destino.

En particular, los datos de la caracterización de los operadores turísticos (Tabla 2) revelan que cuatro de ellos (APS, MC, GM, M) utilizan la reserva natural como servicio turístico; tres de ellos ofrecen servicios gastronómicos (MC, GM y M) que incluyen preparación y venta de alimentos, cenas con productos de sus parcelas (hortalizas, frutas, aromáticas...), también productos procesados como lácteos, amasijos, huevos, además de recorridos por las huertas, degustación de productos y venta de sus productos; dos realizan caminatas ecológicas por senderos naturales y culturales (APS y M); dos incluyen avistamiento de aves y alojamiento de camping (APS y M); y finalmente solo uno ofrece actividades de finca como siembra en la huerta, la ruta de la lana y cuidado de animales de granja.

Tabla 2. Servicios ofrecidos por los operadores turísticos

\begin{tabular}{|c|c|c|c|c|c|c|}
\hline $\begin{array}{l}\text { Prestador } \\
\text { turístico }\end{array}$ & $\begin{array}{c}\text { Reserva } \\
\text { natural }\end{array}$ & Gastronomía & $\begin{array}{l}\text { Caminatas } \\
\text { ecológicas }\end{array}$ & $\begin{array}{c}\text { Avistamiento } \\
\text { de aves }\end{array}$ & $\begin{array}{c}\text { Alojamiento } \\
\text { camping }\end{array}$ & $\begin{array}{c}\text { Actividades } \\
\text { de finca }\end{array}$ \\
\hline $\begin{array}{l}\text { Agroparque } \\
\text { Los Soches }\end{array}$ & $\mathrm{x}$ & & $\mathrm{x}$ & $\mathrm{x}$ & $\mathrm{x}$ & \\
\hline $\begin{array}{l}\text { Mujeres } \\
\text { Chucuas }\end{array}$ & $\mathrm{x}$ & $\mathrm{x}$ & & & & \\
\hline $\begin{array}{c}\text { La Granja de } \\
\text { Mary }\end{array}$ & $\mathrm{x}$ & $\mathrm{x}$ & & & & $\mathrm{x}$ \\
\hline MUTAR & $\mathrm{x}$ & $\mathrm{x}$ & $\mathrm{x}$ & $\mathrm{x}$ & $\mathrm{x}$ & \\
\hline
\end{tabular}

Fuente: elaboración propia. 
Al final, se reitera la conexión que existe entre los cuatro operadores turísticos con su esencia como parte del turismo comunitario. Se evidencia que cada emprendimiento enfoca su propósito turístico con una intensión de promoción del patrimonio cultural y natural de gestión, de generación de recursos económicos para el desarrollo de su comunidad y de defensa de su territorio (Maldonado, 2005; Cardona y Burgos, 2015).

Para el segundo paso, la identificación y comparación de las normas según las características de los prestadores de servicio turístico, cuando se revisaron las resoluciones correspondientes a los protocolos de bioseguridad COVID-19, se identificaron: la norma general (Resolución 666-R666), las específicas del sector turístico (Resoluciones 576 y 1443) y las resoluciones relacionadas con los servicios ofertados por los prestadores de servicios (Resoluciones 749, 1050, 1285 y 1421) (Tabla 3).

Posteriormente, al establecer la relación entre los prestadores de servicios, los servicios ofrecidos y las correspondientes resoluciones (Figura 1), se garantizó la identificación de todos los requerimientos de bioseguridad COVID-19 por parte de cada una de las normas vinculadas a cada uno de los servicios del operador. Es decir, todos los servicios debían contemplar los requerimientos de la resolución 666, de la R1443 y de la R576. El tema de alojamiento estaba registrado en todas las resoluciones del sector turístico; las especificaciones para la prestación de servicio de alimentos se encontraron en dos resoluciones: la R749 y la R1050; y tanto las caminatas ecológicas como las actividades de finca en la R1421.

Tabla 3. Resoluciones bioseguridad COVID-19 del sector turismo

\begin{tabular}{|c|c|}
\hline Norma & Descripción \\
\hline $\begin{array}{l}\text { Resolución } 666 \text { del } \\
24 \text { de abril de } 2020 .\end{array}$ & $\begin{array}{l}\text { Por medio del cual se adopta el protocolo general de bioseguridad (Diario } \\
\text { Oficial No. } 51295 \text { del 24/04/20). }\end{array}$ \\
\hline $\begin{array}{l}\text { Resolución } 749 \text { del } \\
13 \text { de mayo de } 2020 .\end{array}$ & $\begin{array}{l}\text { Protocolo de bioseguridad para el comercio al por mayor y al por menor de } \\
\text { alimentos, bebidas y tabaco, en establecimientos no especializados, así como } \\
\text { el alojamiento en hoteles y actividades de restaurantes, cafeterías y servicio } \\
\text { móvil de comidas (Diario Oficial No.51321 d e21/05/2020). }\end{array}$ \\
\hline $\begin{array}{l}\text { Resolución } 576 \text { de } \\
\text { mayo } 2020 .\end{array}$ & $\begin{array}{l}\text { Sello de calidad "Check in Certificado"." (Ministerio de Comercio, Industria y } \\
\text { Turismo, 2020). }\end{array}$ \\
\hline $\begin{array}{l}\text { Resolución } 1050 \text { del } \\
26 \text { de junio de } 2020 .\end{array}$ & $\begin{array}{l}\text { Protocolo de bioseguridad para el servicio de "entrega para llevar", para las } \\
\text { actividades de expendio a la mesa de comidas preparadas (CIIU 5611); otros } \\
\text { tipos de expendio de comidas preparadas n.c.p. (CIIU 5619) (Diario Oficial } \\
\mathrm{N}^{\circ} 51.360 \text {, del } 29 / 06 / 2020 \text {.) }\end{array}$ \\
\hline $\begin{array}{l}\text { Resolución } 1285 \text { del } \\
29 \text { de julio } 2020 .\end{array}$ & $\begin{array}{l}\text { Protocolo de bioseguridad para los servicios y actividades de alojamiento rural } \\
\text { (CIIU 5514); otros tipos de alojamiento para visitantes (CIUU 5519); } \\
\text { actividades de zonas de camping y parques para vehículos recreacionales } \\
\text { (CIIU 5520) (Diario Oficial No. 51.360, del 29/06/2020). }\end{array}$ \\
\hline $\begin{array}{l}\text { Resolución } 1421 \text { de } \\
\text { agosto } 2020 .\end{array}$ & $\begin{array}{l}\text { Protocolo de bioseguridad para las actividades de los parques de diversión, } \\
\text { jardines botánicos y reservas naturales (Diario Oficial No. } 51413 \text { del } \\
21 / 08 / 2020 \text { ). }\end{array}$ \\
\hline $\begin{array}{l}\text { Resolución } 1443 \\
\text { agosto } 2020 .\end{array}$ & $\begin{array}{l}\text { Protocolo de bioseguridad para las actividades de los operadores turísticos y } \\
\text { en los servicios turísticos prestados en las áreas y atractivos turísticos (Diario } \\
\text { Oficial No. } 51416 \text { del 24/08/2020). }\end{array}$ \\
\hline
\end{tabular}

Fuente: elaboración propia. 
Con lo anterior se evidenció que era importante revisar más de una norma para poder encontrar los detalles de la bioseguridad del COVID-19, necesarios para cubrir los diferentes servicios y buenas prácticas.

En el tercer paso, se realizó la comparación de las diferentes normas de bioseguridad para los cuatro operadores turísticos (Figura 1). En este punto, tanto las investigadoras como los prestadores de servicios al cotejar todos los aspectos que abordan las normas revisadas, lograron reunir en cinco puntos las consideraciones pertinentes e indispensables para una sola estructura: las generalidades de las buenas prácticas para el protocolo, las medidas preventivas y locativas, los diferentes protocolos $\mathrm{o}$ procedimientos clave, el plan de contingencia y riesgos, al igual que el plan de comunicación (Figura 2).

Figura 1. Relación y comparación de los operadores turísticos, los servicios que prestan y las normas de bioseguridad COVID-19 que los incluyen

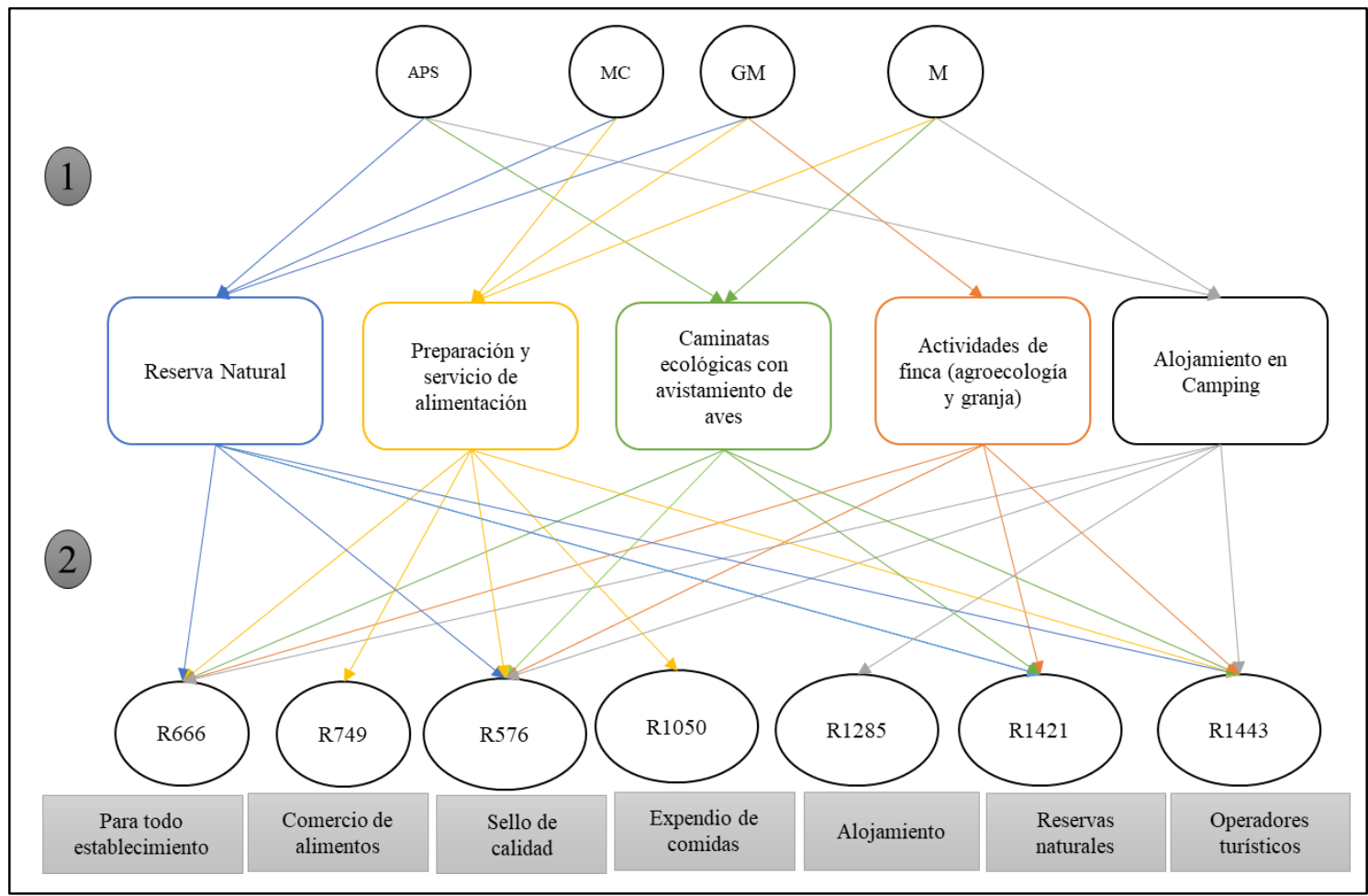

Fuente: elaboración propia. Notas: 1. La relación entre los operadores turísticos del caso (APS, MC, GM y M) y los servicios ofrecidos; 2. La relación entre los servicios ofrecidos y las normas de bioseguridad relacionadas con el COVID-19.

Del mismo modo, al hacer visibles los cinco puntos clave, emergieron los aspectos requeridos para cada uno, logrando co-contruir entre la comunidad y las investigadoras una estructura y un contenido con todas las prácticas de bioseguridad COVID-19 necesarias para los cuatro operadores (Figura 2).

En voz de los representantes, "fue de gran aprendizaje el ejercicio y muy revelador frente a todos los aspectos de bioseguridad y de las buenas prácticas que deben tener en cuenta para atender a todo su público y evitar el contagio por COVID-19, entre otros" (Comunicación de los representantes, noviembre de 2020). Además, manifestaron la 
importancia de ver en detalle las necesidades que tienen que cubrir para la implementación del protocolo y para poder abrir al público de manera adecuada y formal.

En este mismo sentido del aprendizaje, se considera de gran relevancia el que los operadores turísticos cuenten con un protocolo de buenas prácticas de bioseguridad COVID-19 como aporte de la institución educativa para poder dar un paso hacia la certificación "Check in certificado COVID-19 Bioseguro" y así poder acceder a convocatorias y a otros públicos.

Figura 2. Estructura detallada del protocolo de buenas prácticas de bioseguridad COVID-19 para los cuatro operadores turísticos

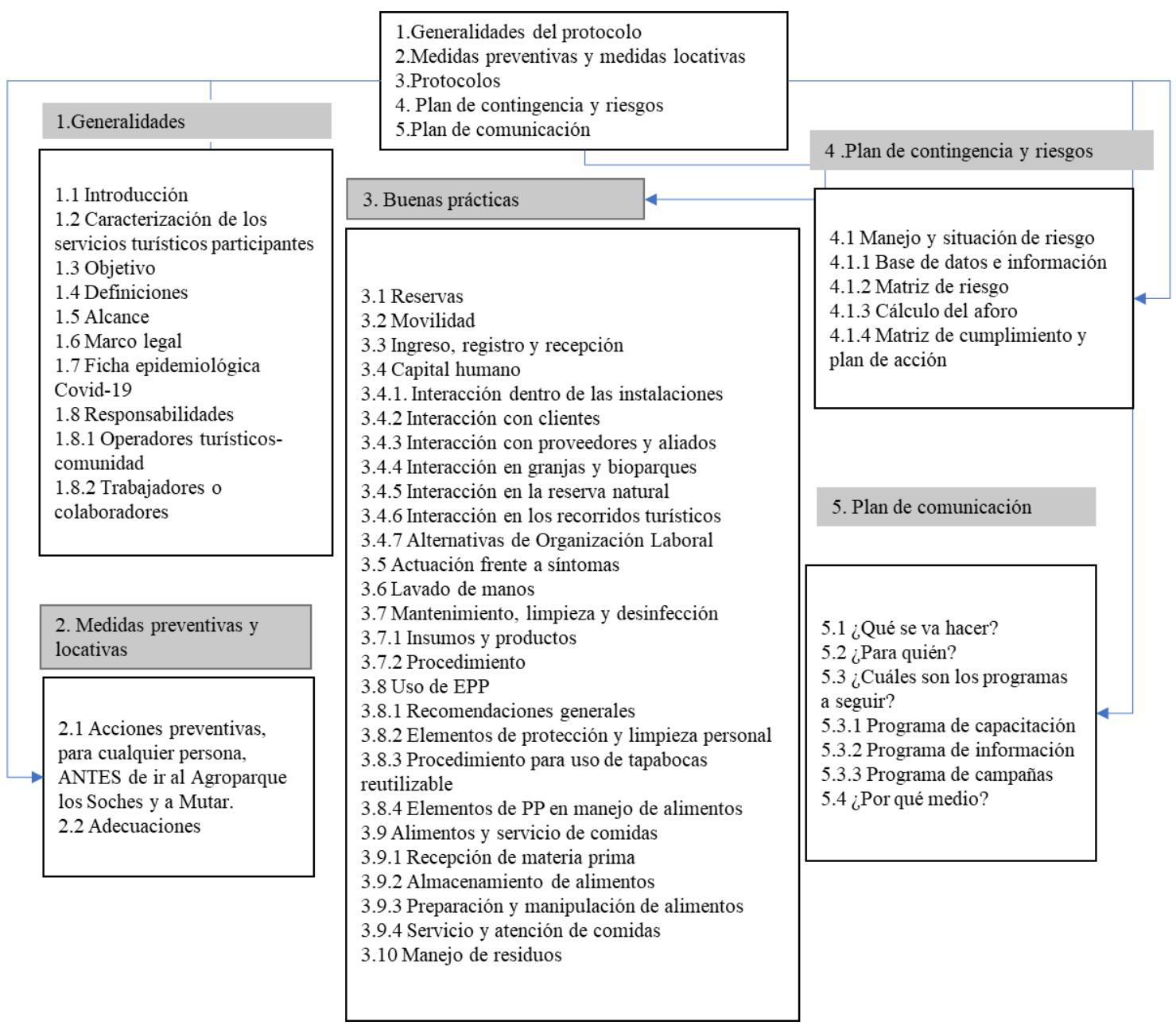

Fuente: elaboración propia.

\section{CONCLUSIONES}

La buena práctica de la bioseguridad es un tema complejo y poco abordado por los operadores turísticos de las comunidades rurales, en este caso de la localidad de Usme en Bogotá-Colombia. Asunto que requiere de trabajo articulado entre los prestadores de servicios y las autoridades locales que regulan la legislación.

Los prestadores de servicio turístico de esta localidad, como parte del turismo comunitario, tienen características ambientales, socioculturales y socioeconómicas 
similares, además de compartir servicios semejantes o complementarios. Lo anterior permitió elaborar un solo protocolo de buenas prácticas de bioseguridad COVID-19 para responder a los requerimientos normativos.

Para la elaboración de un protocolo de buenas prácticas de bioseguridad se requiere conocer la normativa vigente en cualquier parte del mundo. En este estudio la resolución 666 de 2020, fue la medida nacional adoptada para el protocolo general de buenas prácticas de bioseguridad COVID-19 en el sector del turismo en Colombia. Esta debió ser complementada con las resoluciones $1443,1421,1285,1050$ y 749 , todas del 2020, al contar con servicios turísticos de: reserva natural, realización de caminatas ecológicas, avistamiento de aves, actividades de finca, gastronomía y alojamiento tipo camping.

Para la elaboración de un protocolo de buenas prácticas de bioseguridad en coconstrucción con cuatro operadores turísticos, que hacen parte del turismo comunitario de la misma región se pudo: caracterizar a los diferentes operadores e identificar los servicios semejantes y diferentes; indagar y cotejar las normas correspondientes a los productos y servicios que ofrecen; y co-construir entre todos una sola estructura de protocolo para abordar los puntos necesarios para las buenas prácticas de bioseguridad en el antes, durante y después de la operación con los visitantes.

Así, un protocolo de buenas prácticas de bioseguridad COVID-19 que cumple con todos los requerimientos legales en el turismo comunitario de la localidad de Usme, debe incluir las generalidades de los protocolos, las medidas preventivas y locativas según el contexto, los protocolos reglamentarios tanto generales como específicos para el sector turístico, el plan de contingencia y de riesgos, al igual que un plan de comunicación completo que permita divulgar la información correspondiente a toda la comunidad.

La experiencia lograda con este estudio, queda como un ejemplo para la construcción de un documento técnico de buenas prácticas de bioseguridad para que los operadores turísticos, como los de este caso, puedan presentar a las autoridades locales y de esta manera poder reactivar sus servicios. Igualmente es una contribución para que los operadores turísticos puedan acceder a convocatorias actuales dentro del turismo comunitario.

De esta forma, el logro obtenido en este estudio hace parte del conocimiento para las buenas prácticas de bioseguridad en el sector del turismo, en una coyuntura mundial de pandemia, que exigió que todos los sectores entraran en una dinámica de cambio y de atención frente a la bioseguridad, la cual resulta pertinente para mejorar el servicio del turismo comunitario, que justamente tiene limitaciones de presupuesto, planeación, organización y acompañamiento.

\section{REFERENCIAS}

Alcaldía Mayor de Bogotá (2010). Localidad de Usme. Diagnóstico Local con Participación Social 2009-2010. Recuperado de: http://www.saludcapital.gov.co/sitios/VigilanciaSaludPublica/Diagnosticos\%20L ocales/05-USME.pdf (13/05/2021).

Asociación Colombiana de Agencias de Viaje y Turismo 2020. Protocolos de Bioseguridad para el sector del turismo. Recuperado de: https://anato.org/turismocolombia/ (13/05/2021). 
Cardona Prieto, M., y Burgos Doria, R. (2015). El turismo comunitario en Colombia: iniciativa de desarrollo local y estrategia de empoderamiento del patrimonio cultural. Administración y Desarrollo, 45(1). Recuperado de https://dialnet.unirioja.es/servlet/articulo?codigo=6403410 (13/05/2021).

Ceballos-Herrera, F. A (2009). El informe de investigación con estudio de casos. Magis. Revista Internacional de Investigación en Educación, 1(2) ,413-423. Recuperado de: https://www.redalyc.org/pdf/2810/281021548015.pdf (13/05/2021).

Decreto 1443 de Julio 2014. Ministerio de Trabajo. Recuperado de: https://www.mintrabajo.gov.co/documents/20147/36482/decreto_1443_sgsss.pdf/ ac41ab70-e369-9990-c6f4-1774e8d9a5fa (13/05/2021).

Hernández-Sampieri, R., Fernández-Collado, C., y Baptista-Lucio, P. (2014). Desarrollo de la perspectiva teórica: Concepción o elección del diseño de investigación. En R. Hernández-Sampiere (ed.), Metodología de la Investigación (pp. 126-164). México: McGraw-Hill.

Instituto Distrital de Turismo. (2020). SELLO DE BIOSEGURIDAD CHECK - IN CERTIFICADO. Recuperado de: https://www.idt.gov.co/es/sello-check-certificado $(13 / 05 / 2021)$.

Maldonado, C. (2005). Pautas metodológicas para el análisis de experiencias de turismo comunitario. Documento de trabajo No. 73. Serie Red de Turismo Sostenible Comunitario para América Latina (REDTURS). Recuperado de httpds://oit.org/wcmsp5/groups/public/---ed_emp/---emp_ent/--ifp_seed/documents/publication/wcms_117525.pdf (13/05/2021).

Ministerio de Comercio Industria y Turismo (2012). Lineamientos de política para el desarrollo del turismo comunitario en Colombia. Recuperado de: https://www.mincit.gov.co/CMSPages/GetFile.aspx?guid=58fc480a-7a27-4420aac4-e72c8bcee437 (13/05/2021).

Ministerio de Comercio, Industria y Turismo (2020). Resolución 576 de mayo 2020. Recuperado de: https://www.mincit.gov.co/getattachment/26c05e53-a19d-4c12a783-3ca7381a7b71/Resolucion-0576-del-27-de-mayo-de-2020-por-medio-d.aspx $(13 / 05 / 2021)$.

Ministerio de Salud y Protección Social (2020). Resolución 1050 del 26 de junio de 2020. Diario Oficial $N^{\circ}$ 51.360, del 29/06/2020. Recuperado de: https://www.minsalud.gov.co/Normatividad_Nuevo/Resoluci\%C3\%B3n\%20No. \%201050\%20de\%202020.pdf (13/05/2021).

Ministerio de Salud y Protección Social (2020). Resolución 1285 del 29 de julio de 2020.Diario Oficial No. 51390 del 29/07/2020. Recuperado de: https://www.minsalud.gov.co/Normatividad_Nuevo/Resoluci\%C3\%B3n\%20No. \%201285\%20de\%202020.pdf (13/05/2021).

Ministerio de Salud y Protección Social (2020). Resolución 1421 de agosto de 2020. Diario Oficial No. 51413 del 21/08/2020. https://www.minsalud.gov.co/Normatividad_Nuevo/Resoluci\%C3\%B3n\%20No. \%201421\%20de\%202020.pdf (13/05/2021).

Ministerio de Salud y Protección Social (2020). Resolución 1443 del 24 de agosto de 2020. Diario Oficial No. 51416 del 24/08/2020.Recuperada de: 
https://www.minsalud.gov.co/Normatividad_Nuevo/Resoluci\%C3\%B3n\%20No. \%201443\%20de\%202020.pdf (13/05/2021).

Ministerio de Salud y Protección Social (2020). Resolución 749 del 13 de mayo de 2020. Diario Oficial No. 51321 del 21/05/2020. Recuperado de: https://www.minsalud.gov.co/Normatividad_Nuevo/Resoluci\%C3\%B3n\%20No. $\% 20749 \% 20 \mathrm{de} \% 202020 . \operatorname{pdf}(13 / 05 / 2021)$.

Ministerio de Salud y Protección Social de Colombia (2020). Resolución 666 del 24 de abril de 2020. DIARIO OFICIAL 51295 DEL 24/04/20. Recuperado de: https://www.minsalud.gov.co/Normatividad_Nuevo/Resoluci\%C3\%B3n\%20No.

\%20666\%20de\%202020.pdf (13/05/2021).

Organización Mundial de Turismo (S.f). Turismo Rural. Recuperado de: https://www.unwto.org/es/turismo-rural (13/05/2021).

Organización Mundial del Turismo (2020). COVID-19 Respuesta. Recuperado de: https://www.unwto.org/es/turismo-covid-19 (13/05/2021).

Politécnico Internacional (2020). Programa de Bioseguridad COVID-19. Recuperado de: https://politecnicointernacional.edu.co/sw/sites/default/files/2020-

06/PLANBIOSEGURIDADFINAL_compressed.pdf (13/05/2021).

Stake, R. E. (1998). Investigación con estudio de casos. Morata. Madrid.

Yin, R. (1985). Investigación sobre estudio de casos. Diseño y Métodos. SAGE Publications.

Recuperado

de: https://panel.inkuba.com/sites/2/archivos/YIN\%20ROBERT\%20.pdf (13/05/2021). 